\title{
Evaluation of methacholine dose-response curves by linear and exponential mathematical models: goodness-of-fit and validity of extrapolation
}

\author{
G. Verlato*, I. Cerveri**, A. Villani*, M. Pasquetto*, M. Ferrari ${ }^{+}$, \\ F. Fanfulla**, E. Zanolin*, B. Rijcken++, R. de Marco*
}

\begin{abstract}
Evaluation of methacholine dose-response curves by linear and exponential mathematical models: goodness-of-fit and validity of extrapolation. G. Verlato, I. Cerveri, A. Villani, M. Pasquetto, M. Ferrari, F. Fanfulla, E. Zanolin, B. Rijcken, R. de Marco. CERS Journals 1996

ABSTRACT: Several models have been proposed to analyse dose-response curves recorded in bronchoprovocation challenge tests. The aims of the present work were: 1) to investigate which model (linear vs exponential) and which minimization method (trials and errors $v s$ Levenberg-Marquardt) gives better results in terms of data interpolation (goodness-of-fit); and 2) to verify the validity of extrapolation by comparing forced expiratory volume in one second (FEV1) observed after $4 \mathrm{mg}$ methacholine with values extrapolated after truncation of the curves at $2 \mathrm{mg}$.

For these purposes, methacholine dose-response curves were obtained in 832 subjects from a random population sample, as part of the European Community Respiratory Health Survey (ECRHS) in Italy. Methacholine was inhaled up to a maximum dose of $6 \mathrm{mg}$ by dosimeter technique.

The coefficient of determination $\left(\mathbf{r}^{2}\right)$ was significantly higher with the exponential model $(0.81 \pm 0.22$; mean \pm SD) than with the linear model $(0.69 \pm 0.27)$. With both models, extrapolated values were usually lower than observed values. As a consequence, a $20 \%$ fall in FEV1 with respect to postsaline FEV1 was observed in only $24 \%$ and $21 \%$ of the tests, where a $20 \%$ fall had been predicted, respectively, according to the linear and the exponential model.

In conclusion, exponential models are better than linear models with respect to data interpolation of methacholine dose-response curves. However, they are worse with respect to extrapolation to higher doses. With any model, extrapolation of dose-response curves by one doubling-dose should be avoided.

Eur Respir J., 1996, 9, 506-511.
\end{abstract}

*Cattedra di Statistica Medica e Biometria, Istituto di Igiene, and +Istituto di Semeiotica e Nefrologia Medica, Università di Verona, Policlinico di Borgo Roma, Verona, Italy. ** Istituto di Tisiologia e Malattie dell 'Apparato Respiratorio, Università di Pavia, Policlinico S. Matteo, Pavia, Italy. ${ }^{++}$Department of Epidemiology, State University of Groningen, Groningen, The Netherlands.

Correspondence: G. Verlato

Cattedra di Statistica Medica e Biometria Istituto di Igiene

Università di Verona

Policlinico di Borgo Roma

Via delle Menegone

37134 Verona

Italy

Keywords: Bronchial provocation tests, forced expiratory volume, log dose-response relationship, methacholine, model fit

Received: March 271995

Accepted after revision October 301995

The results presented here are from a local analysis of data collected for the European Community Respiratory Health Survey (ECRHS). Any final international comparison may use a different form of analysis.
The scientific community is still debating how to express bronchial responsiveness [1]. In particular, studies on bronchial reactivity and its relationship with asthma and other chronic obstructive pulmonary diseases have to face the difficult problem of so-called censored data of bronchoprovocation challenge tests. This problem is larger in epidemiological studies than in the clinical context: firstly, the maximal dose of bronchoconstrictor agent which can be administered is limited by safety considerations and side-effects; secondly, most subjects are not asthmatics and, thus, present a low bronchial reactivity. The resulting information loss limits the possibilities for statistical analyses [2, 3]. The problem of censored data becomes even more important in longitudinal studies, having to deal with changes in the estimates of censored data [4-7].

To overcome this problem, several techniques have been proposed: new indices linked to a smaller fall of forced expiratory volume in one second (FEV1) (i.e. 6,
10 and 15\%); extrapolations to one doubling-dose beyond the maximum dose administered; area under dose-response curve; dose-response slope [3, 8-14].

Recently CHINN et al. [15] proposed estimating the provocative dose of agonist required to cause a $20 \%$ fall in FEV1 (PD20) by fitting an exponential curve to the observed data and by extrapolating by one doublingdose. They compared extrapolated PD20 with two alternative measures of response, two-point slope [13] and least-squares slope [2], and concluded that PD20 is somewhat more suitable. Indeed this method presents several advantages: its results are repeatable; it allows discrimination between asthmatic and nonasthmatic subjects; and it can be synthesized through variables which satisfy the main requirements for statistical analysis, i.e. normality and stability of variance. However, the validity of extrapolation has not yet been verified through a direct comparison of observed and expected data [16]. 
In the three Italian centres participating in the European Community Respiratory Health Survey (ECRHS) [17, 18], the protocol of the methacholine challenge test was slightly modified by administering at least one additional dose of methacholine (4 mg cumulative dose), without impairing safety conditions [19-21]. This gave the opportunity to verify the validity of extrapolation by one doubling-dose in the usual range of epidemiological studies.

The aims of the present work were: 1) to investigate which model (linear [2] vs exponential [15]) and which minimization method (trials and errors [22] vs LevenbergMarquardt [23]) gives better results in terms of data interpolation (goodness-of-fit); 2) to verify the validity of extrapolation by comparing FEV1 observed after $4 \mathrm{mg}$ methacholine with values extrapolated after truncation of the dose-response curve at $2 \mathrm{mg}$.

\section{Material and methods}

\section{Subjects}

The design of the ECRHS [17] involved two stages. In Stage I, a screening questionnaire on respiratory symptoms was mailed to a probability sample of 20-44 year old males and females resident in the areas. In the second stage, a 20\% random sample of responders to the mailed questionnaire was invited to the local chest clinic, in order to undergo a standardized clinical interview, lung function tests, including a bronchial challenge with methacholine, and allergen skin tests. In addition, people who, in the screening questionnaire, had reported asthma attacks, taking medicines for asthma, or awakening due to an attack of shortness of breath, were asked to attend Stage II.

In this way, 1,835 sujects out of 6,031 who had previously returned the questionnaire were invited to the clinical centres. Of these, 899 agreed to participate in stage II, yielding an attendance rate of 49\% [24]. An additional 190 subjects who declared asthma-like symptoms in the screening questionnaire underwent clinical examination. Moreover, 15 subjects who had fulfilled the Stage II protocol in Verona without attending Stage I were admitted to the study, yielding an overall number of 1,104 subjects.

Of these 1,104 subjects, 850 performed technically satisfactory methacholine challenge tests. After eliminating 18 subjects receiving just one or two doses of methacholine, the study base comprised 832 subjects. Informed consent was given by each subject participating in the study.

\section{Methacholine bronchial challenge}

Bronchial responsiveness was measured by methacholine challenge. For the administration of methacholine Mefar MB3 Dosimeters (Mefar spa, Bovezzi, Italy) were used.
Lung volumes (forced vital capacity (FVC) and FEV1) were measured by means of Biomedin Spirometer (Biomedin srl, Padova, Italy) taking the best of five satisfactory manoeuvres. A solution from lyophilized methacholine chloride (Provocoline, Hoffman La Roche, Basel, Switzerland) was used as a provocative agent.

Inclusion criteria, measurement conditions and laboratory protocol are reported in the international protocol $[17,18]$. Briefly, all subjects reporting a heart attack in the last 3 months or any other disease, affected by epilepsy requiring medical treatment, pregnant or breast-feeding, or taking beta-blockers for any reason, were excluded from the challenge test. In addition, methacholine challenge was not performed when the subjects had difficulty in complying with instructions or had a best initial FEV1 less than $70 \%$ predicted or a best postdiluent FEV1 less than $90 \%$ of initial FEV1.

According to the national protocol, the test was stopped when FEV1 had fallen by $20 \%$ or more with respect to postsaline FEV1, when a cumulative dose of $6 \mathrm{mg}$ methacholine had been administered (while the European protocol stopped at $2 \mathrm{mg}$ ), or when the subject refused to continue or was not able to perform two technically satisfactory manoeuvres out of five attempts. The following cumulative doses were given: $0.0156,0.0625,0.25,1$, $2,4,6 \mathrm{mg}$. If the subject had a past history of asthma or wheezing, a more careful protocol was adopted by starting from $0.00185 \mathrm{mg}$ and progressively doubling the cumulative dose. In one centre (Verona), the dose of 6 $\mathrm{mg}$ was replaced by a dose of $8 \mathrm{mg}$.

In order to standardize the administration methods, all the Italian research teams underwent a training session and all the tests were conducted using the same equipment. Nebulizers, of known output, were checked prior and during each challenge.

Bronchoconstriction was readily reversed by administration of salbutamol via metered-dose inhaler. No noticeable adverse reactions were observed at a cumulative dose of 6 or $8 \mathrm{mg}$ methacholine.

\section{Mathematical models}

The two-point slope [13] was computed as the percentage decline in FEV1 from the postsaline value to that of the total cumulative dose administered, divided by the total dose. The least-squares slope was calculated from all data except the postsaline FEV1 [2].

The exponential model [11]:

$$
\mathrm{FEV} 1=c-\exp \left(\mathrm{a}+\mathrm{b}\left(\log _{10} \text { dose }\right)\right)
$$

was fitted to all data (including postsaline FEV1) using two different minimization methods, the trials and errors [22] and Levenberg-Marquardt method [23]. To include postsaline FEV1 in the model, it was necessary to assume that the corresponding dose of methacholine was higher than zero $(0.0001 \mathrm{mg})$. The models are presented in graphic form in figure 1.

In a few cases, the temporary estimate of $c$ tended to increase enormously during the minimization process, 
a)

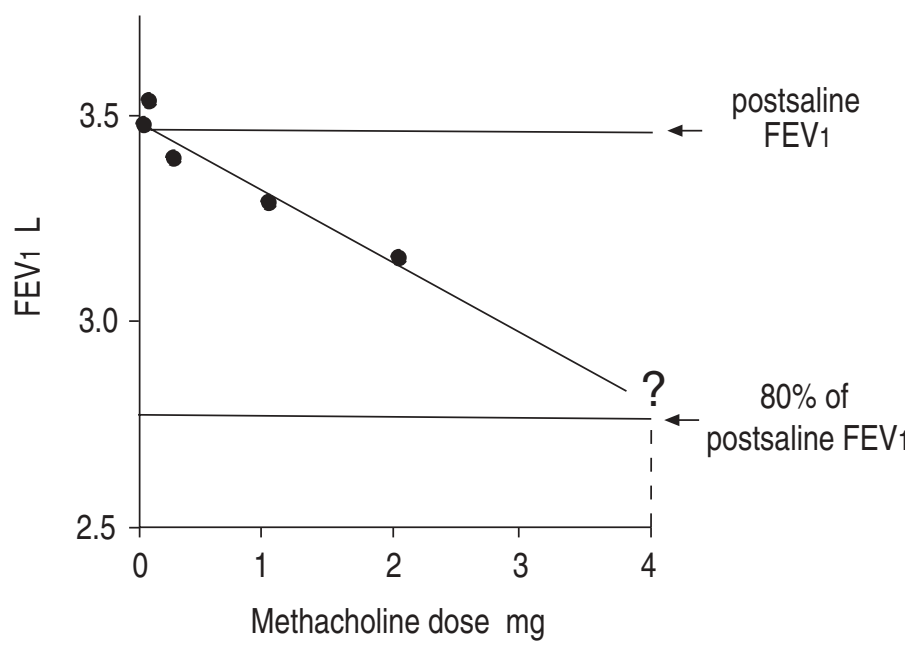

b)

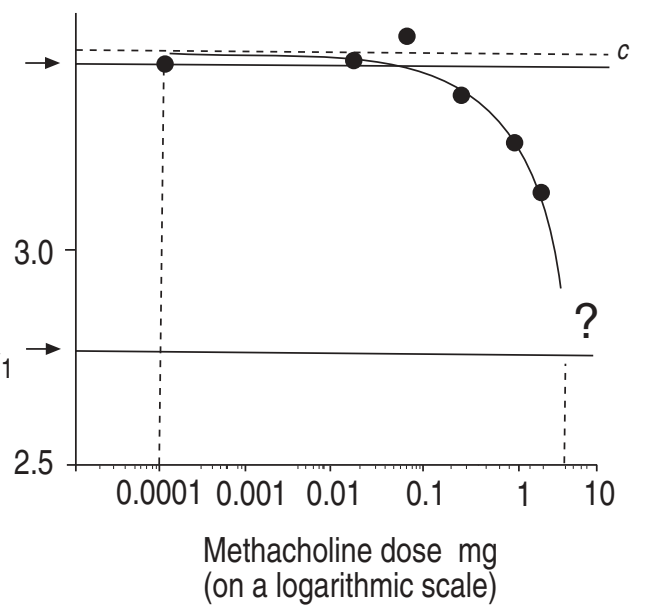

Fig. 1. - Graphic representation of: a) a linear model; and b) an exponential model, fitted to methacholine dose-response curve truncated at 2 $\mathrm{mg}$. The question mark stands for extrapolation at $4 \mathrm{mg}$ methacholine. Postsaline FEV 1 was not considered for the linear model, whilst it was taken into account for the exponential model, assuming a corresponding methacholine dose of $0.0001 \mathrm{mg}$. The value of $c$, the asymptote of the exponential model: $\mathrm{FEV}_{1}=c-\exp \left(\mathrm{a}+\mathrm{b}\left(\log _{10}\right.\right.$ dose $\left.)\right)$, is also shown. $\mathrm{FEV} 1$ : forced expiratory volume in one second.

yielding unphysiological values of $c$ (up to $100,000 \mathrm{~L} \cdot \mathrm{s}^{-1}$ ) and turning the exponential model into a linear one. To prevent this outcome, $c$ was not allowed to increase more than $30 \%$ with respect to postsaline FEV1. This problem did not occur with linear models, since the intercept of the least-squares regression never differed from postsaline FEV1 by more than $18 \%$.

A QBASIC program and a Mathematica program, the former modified (V.G.) the latter developed in our laboratory (V.A. and P.M), were used to fit the exponential equation to the experimental data by the method of trials and errors and by the method by LevenbergMarquardt, respectively. This software is available from the Correspondence author, address on first page of paper.

Truncation of the curves. The goodness-of-fit of different methods was studied for all of the 832 subjects, whilst the validity of the extrapolation was investigated for the 639 subjects who were administered a cumulative dose of $2 \mathrm{mg}$ methacholine without presenting a 20\% fall in FEV1, and thus received an additional dose of $4 \mathrm{mg}$ methacholine. Extrapolated values lower than zero were recoded at zero.

\section{Statistical analysis}

Data are presented as mean \pm SD. The coefficient of determination $\left(\mathrm{r}^{2}\right)$ was used to evaluate the goodnessof-fit of linear versus exponential models. Since this measure was not normally distributed according to the Kolmogorov-Smirnov test, Friedman's nonparametric twoway analysis of variance was used to compare coefficients of determination obtained with different models.

The relationship between observed and extrapolated values of FEV1 at $4 \mathrm{mg}$ methacholine was studied by a simple linear regression. A t-test was used to assess whether the slope of the regression line was significantly different from that of the identity line [25].

\section{Results}

Of the total 832 subjects given at least three doses of methacholine, 131 presented a $20 \%$ fall in FEV1 below $4 \mathrm{mg}$ methacholine, and 35 at $4 \mathrm{mg}$. The number of doses administered (mean $\pm \mathrm{SD}$ ) was 7.0 \pm 1.8 (range 3-14).

\section{Data interpolation}

Both linear and exponential models could be fitted to all 832 curves. The $\mathrm{r}^{2}$ was significantly lower $(\mathrm{p}<0.001)$ with the linear model $(0.69 \pm 0.27$ with the least-squares method) than with the exponential model $(0.81 \pm 0.22$ both with trials and errors and Levenberg-Marquardt algorithm).

The linear model slightly underestimated postsaline FEV1, whilst the exponential models slightly overestimated it. The percentage difference between the intercept of the linear regression and the corresponding postsaline FEV1 amounted to $-2.2 \pm 2.9 \%$ (range -18.1 to $+7.8 \%$ ), whilst the percentage difference between the upper asymptote and postsaline FEV1 was $3.0 \pm 7.2 \%$ (range -8.8 to $+30.0 \%$ ) and $1.9 \pm 6.1 \%$ (range -8.8 to $+30.0 \%$ ), respectively, for the trials and errors method and the LevenbergMarquardt algorithm.

When allowing for any $c$ values, exponential models yielded unphysiological asymptotes, exceeding postsaline FEV 1 by $30 \%$ or more, in 65 cases $(8 \%)$ with trials and errors and in 31 cases (4\%) with the Levenberg-Marquardt algorithm.

\section{Data extrapolation}

Truncation of the curves, by omitting doses equal to or higher than $4 \mathrm{mg}$ methacholine, enabled FEV1 extrapolation $(n=639)$. Before extrapolating to $4 \mathrm{mg}$, goodness-of-fit of linear and exponential models on truncated curves was verified through the $\mathrm{r}^{2}$. Again this measure 

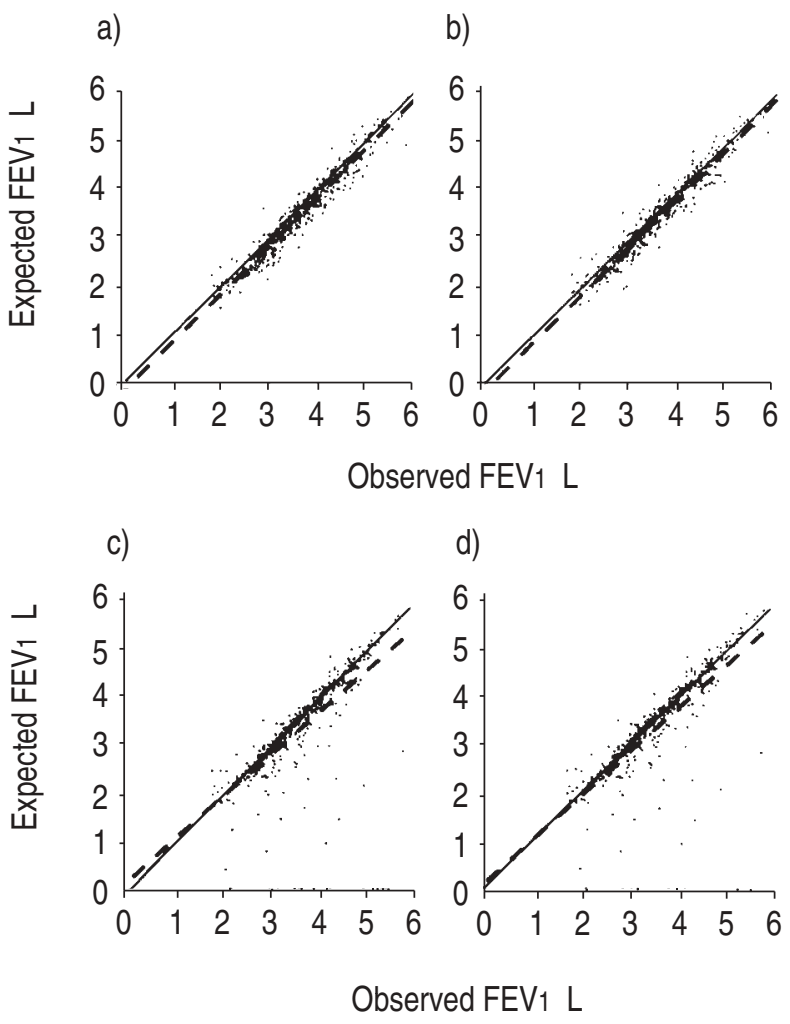

Fig. 2. - Relationship between observed and expected FEV1 at $4 \mathrm{mg}$ methacholine. Expected values were extrapolated from dose-response curves truncated at $2 \mathrm{mg}$ by using four different models. a) Linear model, two-point slope; b) Linear model, least-squares slope; c) Exponential model, trials and errors; d) Exponential model, LevenbergMarquardt algorithm. Solid lines: identity lines; dashed lines: regression lines. FEV1: forced expiratory volume in one second was significantly lower $(\mathrm{p}<0.001)$ with the linear model $(0.64 \pm 0.29$ with the least-squares method) than with the exponential model $(0.74 \pm 0.26$ with trials and errors and $0.73 \pm 0.27$ with Levenberg-Marquardt algorithm).

Figure 2 shows the FEV1 extrapolated to $4 \mathrm{mg}$ (exp) versus the experimentally determined FEV1 (obs). The regression line was closer to the identity line with linear models (exp $=-0.15+1.00 *$ obs for two-point slope (fig. 2a) and exp=-0.16+1.01*obs for least-squares slope (fig. 2b)) than with exponential models (exp $=0.24+0.87 *$ obs for trials and errors (fig. 2c) and exp=0.10+0.92*obs with Levenberg-Marquardt algorithm (fig. 2d)). Indeed, the slope of the regression line was significantly different from unit with exponential models but not with linear ones. The linear correlation coefficient indicated a highly significant relationship for linear models ( $r=0.97$ for both two-point slope and least-squares slope) and a much weaker relationship for exponential models $(r=0.70$ for trials and errors and $\mathrm{r}=0.76$ for Levenberg-Marquardt method).

The deviation of the extrapolated values in percentage of the measured values amounted to $-4.5 \pm 6.1 \%,-4.0 \pm 6.2 \%$, $-5.8 \pm 19.2 \%$ and $-5.1 \pm 17.6 \%($ mean $\pm \mathrm{SD})$ when adopting two-point slope, least-squares slope, trials and errors and Levenberg-Marquardt algorithm, respectively.

Extrapolation was also evaluated by recoding FEV1 into a dichotomous variable, where the response was considered "positive" in the presence of a $20 \%$ fall in FEV 1 with respect to postsaline FEV1 and "negative" otherwise (table 1). Specificity, defined as the percentage of predicted negative responses among observed negative responses was similar for all models, ranging 85-87\% (table 2). On the contrary, sensitivity, defined as the

Table 1. - Validity of extrapolation by one doubling-dose, verified through comparison of the bronchial response ${ }^{\dagger}$ observed at $4 \mathrm{mg}$ with the bronchial response extrapolated after truncation of the curve at $2 \mathrm{mg}(\mathrm{n}=639)$

Two-point slope

\begin{tabular}{lcc}
\hline $\begin{array}{l}\text { Observed } \\
\text { response }\end{array}$ & $\begin{array}{c}\text { Observed } \\
\text { nonresponse }\end{array}$ \\
\hline $\begin{array}{l}\text { Expected } \\
\text { response }\end{array}$ & 31 & 91 \\
\hline $\begin{array}{l}\text { Expected } \\
\text { nonresponse }\end{array}$ & 4 & 513 \\
\hline
\end{tabular}

Trials and errors

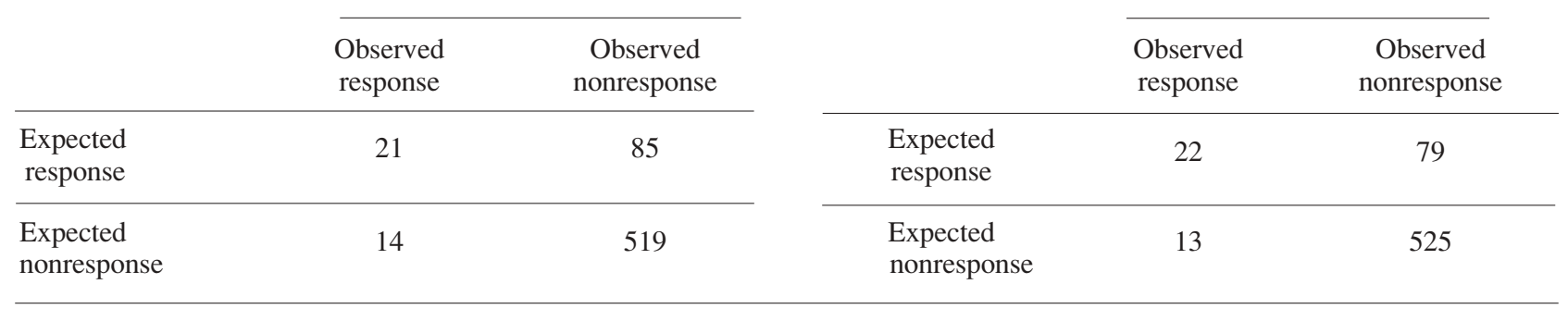

$\dagger$ : Bronchial response was coded as dichotomous variable, where response was considered a decrease in FEV $1 \geq 20 \%$ with respect to postsaline FEV1, whilst all other outcomes were coded as nonresponse. Linear models (two-point slope and least-square slope) are compared with exponential models (trials and errors and Levenberg-Marquardt). FEV1: forced expiratory volume in one second.

Least -squares slope

$\begin{array}{lc}\begin{array}{l}\text { Observed } \\ \text { response }\end{array} & \begin{array}{c}\text { Observed } \\ \text { nonresponse }\end{array}\end{array}$

\begin{tabular}{lcc}
\hline $\begin{array}{l}\text { Expected } \\
\text { response }\end{array}$ & 27 & 87 \\
\hline $\begin{array}{l}\text { Expected } \\
\text { nonresponse }\end{array}$ & 8 & 517 \\
\hline
\end{tabular}

Levenberg-Marquardt 
Table 2. - Validity of extrapolation by one doublingdose, verified through comparison of the bronchial response observed at $4 \mathrm{mg}$ with the bronchial response extrapolated after truncation of the curve at $2 \mathrm{mg}$

\begin{tabular}{lcccc}
\hline & $\begin{array}{c}\text { Sensitivity } \\
\%\end{array}$ & $\begin{array}{c}\text { Specificity } \\
\%\end{array}$ & $\begin{array}{c}+\mathrm{ve} \\
\%\end{array}$ & $\begin{array}{c}-\mathrm{ve} \\
\%\end{array}$ \\
\hline $\begin{array}{l}\text { Two-point } \\
\text { slope }\end{array}$ & 89 & 85 & 25 & 99 \\
$\begin{array}{l}\text { Least-square } \\
\text { slope }\end{array}$ & 77 & 86 & 24 & 98 \\
$\begin{array}{l}\text { Trials and } \\
\text { errors }\end{array}$ & 60 & 86 & 20 & 97 \\
$\begin{array}{l}\text { Levenberg- } \\
\text { Marquardt }\end{array}$ & 63 & 87 & 22 & 98 \\
\hline
\end{tabular}

Sensitivity, specificity, positive (+ve) and negative (-ve) predictive values of prediction of a bronchial response ( $20 \%$ fall in FEV1 with respect to postsaline FEV1) are presented with linear and exponential models. Sensitivity: percentage of expected responses among observed responses; specificity: percentage of expected nonresponses among observed nonresponses; positive predictive values: percentage of observed responses among expected responses; negative predictive value: percentage of observed nonresponses among expected nonresponses. FEV1: forced expiratory volume in one second.

percentage of predicted positive responses among observed positive responses, was higher with linear models ( $89 \%$ for two-point slope and $77 \%$ for least-square slope) than with exponential models $(60 \%$ for trials and errors and $63 \%$ for Levenberg-Marquardt algorithm).

With extrapolation, a larger number of $20 \%$ falls in FEV1 was predicted than actually observed (table 1 ). At $4 \mathrm{mg}$ methacholine, FEV1 fell by $20 \%$ or more with respect to postsaline values in only $25 \%$ (31 of 122), $24 \%$ (27 of 114), 20\% (21 of 106) and $22 \%$ (22 of 101) of the tests, where a $20 \%$ fall was predicted according to two-point slope, least-squares slope, trials and errors and Levenberg-Marquardt algorithm, respectively (table 2 ). The positive predictive values increased only slightly when considering the bronchial response to an additional dose of methacholine (6 or $8 \mathrm{mg}$ ), amounting to $37 \%$ (45 of 122), 37\% (42 of 114), $27 \%$ (29 of 106) and 30\% (30 of 101), respectively, with the different models.

\section{Discussion}

Censored data of dose-response curves represent one of the most important problems in epidemiological studies on bronchial reactivity [1]. Among the several methods proposed to solve this problem, the most popular ones in recent studies are the slope of the dose-response curve, which does not require a threshold fall in functional parameters and, thus, can be computed for almost all subjects [2, 13], and extrapolation to one doublingdose above the maximum dose administered [15].

The aim of the present study was to compare different mathematical models for the dose-response curves, with respect to goodness-of-fit and validity of extrapolation.
As far as the first point is concerned, $\mathrm{r}^{2}$ was higher with exponential models than with linear models, suggesting that, at least in this dose range, the pattern of dose-response curves can be approximated better by an exponential model on a semilogarithmic scale than by a linear model on a normal scale.

It must be pointed out, however, that the two models are equivalent from a mathematical point of view: a linear model turns into an exponential one after log transformation of $x$ values. Thus, the statement that exponential models are better with respect to data interpolation is relevant from a methodological point of view rather than from a physiological one. Indeed, dose-response curves of methacholine challenges are usually reported on semi-logarithmic scale, as for instance when computing PD20 [26, 27].

Both minimization procedures, trials and errors and Levenberg-Marquardt algorithm, allowed the exponential curves to be fitted equally well to the observed data.

As far as extrapolation to one doubling-dose is concerned, to our knowledge, the present study is the first attempt to evaluate extrapolation by one doubling-dose on the basis of the criterion of validity: expected values are directly compared with observed values. Up to now, extrapolation by one doubling-dose has been evaluated mainly on statistical considerations, such as repeatability, normality and stability of variance [15].

Linear models were slightly better than exponential models with respect to extrapolation by one doublingdose (4 mg methacholine). However, in both cases extrapolation underestimated the value of FEV1 which was actually observed. As a consequence, when adopting the $20 \%$ fall in FEV1 as a cut-off, the number of predicted responses was three-times larger than the number of responses actually observed. Moreover, most false positives did not respond even at higher doses (6-8 mg methacholine). Indeed, for this low positive predictive value extrapolation by one doubling-dose is not suited for epidemiological studies dealing with risk factors for bronchial hyperresponsiveness.

A rather straight forward explanation can be given for this result. The complete dose-response curves present a sigmoid shape [14, 16], which is clearly evident in most subjects only when very high doses of methacholine are administered. In epidemiological studies these doses are rarely attained, so that only the first part of the sigmoid curve is recorded, which can be approximated by a straight line or by an exponential curve. Extrapolation by one doubling-dose could have taken place on that part of the curve (2-4 mg methacholine) where the response is progressively decreasing towards the plateau value. This could be the reason why extrapolation by one doubling-dose underestimated FEV1 at $4 \mathrm{mg}$.

These results are further supported by the fact that the study protocol was very strict and rigorous, since it had been specifically designed to prevent possible biases arising from different testing procedures and difference in subject compliance.

As a consequence, if the study design does require that PD20 be assessed in as large as possible a number of subjects, the first attempt to diminish censored data should 
consist in increasing the maximum dose administered. The present study as well as previous ones [9, 19-21] confirm that, even at very high doses of methacholine ( $8 \mathrm{mg}$ cumulative dose), when bronchoconstriction does occur, it can be readily reversed by inhalation of salbutamol via metered-dose inhaler. It must be pointed out that a more careful protocol was used for subjects with a past history of asthma or wheezing. Moreover, no noticeable adverse reactions were observed.

In conclusion, according to the methacholine challenge tests, performed during the ECRHS in Northern Italy, exponential models are better than linear models with respect to data interpolation, but are worse with respect to extrapolation to higher doses. However, with any model the use of extrapolation by one doubling-dose to increase the number of estimates should be discouraged.

\section{Members of ECRHS - Italy}

Università di Verona: R. de Marco (national co-ordinator), V. Lo Cascio (local co-ordinator), C. Campello (principal investigator), F. Rossi, C. Biasin, A. Cannistrà, B. Cenci, E. Destefani, M. Ferrari, M. Girotto, G. Lampronti, C. Martini, M. Olivieri, A. Poli, S. Tardivo, G. Verlato, A. Villani, M.E. Zanolin; Università di Pavia: A. Marinoni (local co-ordinator), I. Cerveri (principal investigator), R. Alesina, O. Basso, L. Berrayah, R. Brusotti, F. Fanfulla, P. Moi, M.C. Zoia; Università di Torino: C. Bucca (principal investigator), C. Romano (principal investigator), M. Aime, A. Cerutti, F. Chiampo, W. Gallo, G. Rola, F. Sulotto; USSL 77-Pavia: L. Casali (principal investigator), C. Frati, P. Karytinos; USSL 7-Torino: M. Bugiani (local co-ordinator), W. Arossa, E. Caria, A. Carosso, G. Castiglioni, P. Piccioni.

Acknowledgements: This study was partially supported by the Italian National Research Council (CNR), targeted project "Prevention and control: disease sectors", grant n. 93.00671.41.

\section{References}

1. Rijcken B, Schouten JP. Measuring bronchial responsiveness in epidemiology. Eur Respir J 1993; 6: 617-618.

2. Abramson MJ, Saunders NA, Hensley MJ. Analysis of bronchial reactivity in epidemiological studies. Thorax 1990; 45: 924-929.

3. Rijcken B, Schouten JP, Weiss ST, Meinesz AF, de Vries $\mathrm{K}$, van der Lende $\mathrm{R}$. The distribution of bronchial responsiveness to histamine in symptomatic and in asymptomatic subjects: a population-based analysis of various indices of responsiveness. Am Rev Respir Dis 1989; 140: 615-623.

4. Britton JR, Burney PGJ, Chinn S, Papacosta AO, Tattersfield AE. The relationship between change in airway reactivity and change in respiratory symptoms and medication in a community study. Am Rev Respir Dis 1988; 138: 530-534.

5. Britton JR, Chinn S, Burney PGJ, Papacosta AO, Tattersfield AE. Seasonal variation in bronchial reactivity in a community population. J Allergy Clin Immunol 1988; 82: 134-139.

6. Vedal S, Enarson DA, Chan H, Ochnio J, Tse KS, ChanYeung MA. A longitudinal study of the occurrence of bronchial hyperresponsiveness in western red cedar workers. Am Rev Respir Dis 1988; 137: 651-655.

7. Peat JK, Unger WR, Combe D. Measuring changes in logarithmic data, with special reference to bronchial responsiveness. J Clin Epidemiol 1994; 47: 1099-1108.
8. Bruschi C, Cerveri I, Zoia MC, Maccarini L, Grassi M, Rampulla C. Bronchial responsiveness to inhaled methacholine in epidemiological studies: comparison of different indices. Eur Respir J 1989; 2: 630-636.

9. Cerveri I, Bruschi C, Zoia MC, et al. Smoking habit and bronchial reactivity in normal subjects: a populationbased study. Am Rev Respir Dis 1989; 140: 191-196.

10. Knox AJ, Coleman HE, Britton JR, Tattersfield AE. A comparison of three measures of the response to inhaled methacholine. Eur Respir J 1989; 2: 736-740.

11. Chinn S, Britton JR, Burney PGJ, Tattersfield AE, Papacosta AO. Estimation and repeatability of the response to inhaled histamine in a community survey. Thorax 1987; 42: 45-52.

12. Forastiere F, Pistelli R, Michelozzi $\mathrm{P}$, et al. Indices of nonspecific bronchial responsiveness in a pediatric population. Chest 1991; 100: 927-934.

13. O'Connor G, Sparrow D, Taylor D, Segal M, Weiss S. Analysis of dose-response curves to methacholine: an approach suitable for population studies. Am Rev Respir Dis 1987; 136: 1412-1417.

14. Woolcock AJ, Salome CM, Yan K. The shape of the dose-response curve to histamine in asthmatic and normal subjects. Am Rev Respir Dis 1984; 130: 71-75.

15. Chinn S, Burney PGJ, Britton JR, Tattersfield AE, Higgins BG. Comparison of PD20 with two alternative measures of response to histamine challenge in epidemiological studies. Eur Respir J 1993; 6: 670-679.

16. Aerts JGJV, Bogaard JM, Overbeek SE, Verbraak AFM, Thio P. Extrapolation of methacholine log-dose response curves with a cumulative Gaussian distribution function. Eur Respir J 1994; 7: 895-900.

17. Burney PGJ, Luczynska C, Chinn S, Jarvis D. The European Community Respiratory Health Survey. Eur Respir J 1994; 7: 954-960.

18. The European Community Respiratory Health Survey. Medicine and Health. European Commission, DirectorateGeneral XIII, Office for Official Publications, L-2920 Luxembourg, 1994.

19. Cerveri I, Bruschi C, Zoia MC, et al. Distribution of bronchial nonspecific reactivity in the general population. Chest 1988; 93: 26-30.

20. Corrao W. Bronchoprovocation challenge: a clinical test whose time has come. Chest 1993; 104: 1323-1324.

21. Pratter MR, Bartter TC, Dubois J. Bronchodilator reversal of bronchospasm and symptoms incurred during methacholine bronchoprovocation challenge: documentation of safety and time course. Chest 1993; 104: 1342-1345.

22. Schiff JD. A simple program in BASIC for least-squares fitting of certain equations to experimental data. Int $J$ Biomed Comput 1985; 16: 143-148.

23. Bazaraa MS, Sherali HD, Shetty CM. In: Nonlinear programming: theory and algorithms. New York, John Wiley and Sons, 1993, pp. 312-315.

24. de Marco R, Verlato G, Zanolin E, Bugiani M, Drane JW. Nonresponse bias in EC Respiratory Health Survey in Italy. Eur Respir J 1994; 7: 2139-2145.

25. Altman DG. In: Practical statistics for medical research. London, Chapman \& Hall, 1991.

26. Juniper EF, Cockcroft DW, Hargreave FE. Histamine and methacholine inhalation tests: tidal breathing method. Laboratory procedure and standardisation. Lund, Sweden, AB Draco, 1991.

27. Sterk PJ, Fabbri LM, Quanjer PhH, et al. Airway responsiveness: standardized challenge testing with pharmacological, physical and sensitizing stimuli in adults. Eur Respir J 1993; 6 (Suppl. 16): 53-83. 